\title{
On Affine Orbifold Nets Associated with Outer Automorphisms
}

\author{
Feng $\mathrm{Xu}^{\star}$ \\ Department of Mathematics, University of California at Riverside, \\ Riverside, CA 92521, USA. E-mail: xufeng@math.ucr.edu
}

Received: 3 November 2008 / Accepted: 6 November 2008

Published online: 5 March 2009 - (C) The Author(s) 2009. This article is published with open access at Springerlink.com

\begin{abstract}
We construct solitons in affine orbifold nets associated with outer automorphisms, and we show that our construction gives all the twisted representations of the fixed point subnet. This allows us to settle a number of questions concerning such orbifold constructions.
\end{abstract}

\section{Introduction}

Let $\mathcal{A}$ be a completely rational conformal net (cf. Section 2.3 and Def. 2.6). Let $\Gamma$ be a finite group acting properly on $\mathcal{A}$ (cf. Definition (2.4)). It is proved in [27] that the fixed point subnet (the orbifold) $\mathcal{A}^{\Gamma}$ is also completely rational, and by [16] $\mathcal{A}^{\Gamma}$ has finitely many irreducible representations which are divided into two classes: the ones that are obtained from the restrictions of a representation of $\mathcal{A}$ to $\mathcal{A}^{\Gamma}$ which are called untwisted representations, and the ones which are twisted (cf. definition after Th. 2.7). It follows from Th. 2.7 that a twisted representation of $\mathcal{A}^{\Gamma}$ always exists if $\mathcal{A}^{\Gamma} \neq \mathcal{A}$. The motivating question for this paper is to construct these twisted representations of $\mathcal{A}^{\Gamma}$.

It turns out that all representations of $\mathcal{A}^{\Gamma}$ are closely related to the solitons of $\mathcal{A}$ (cf. Section 3.3 and Prop. 2.8). Solitons are representations of $\mathcal{A}_{0}$, the restriction of $\mathcal{A}$ to the real line identified with a circle with one point removed. Every representation of $\mathcal{A}$ restricts to a soliton of $\mathcal{A}_{0}$, but not every soliton of $\mathcal{A}_{0}$ can be extended to a representation of $\mathcal{A}$. The construction of the soliton depends on the net $\mathcal{A}$ and the action of $\Gamma$. In this paper we consider the orbifold net associated with $S U(n)_{k}$ with an outer automorphism. When $k=1$, this case is already covered by the general results of [6] and in the framework of Vertex Algebras by [3]. Our work will build upon the results of [6 and 3].

The main difference between the case considered in this paper and those of [14] is that it is not an easy question to determine the index of solitons, hence the strategy of

\footnotetext{
* Supported in part by NSF.
} 
adding up all indices to see if it agrees with the index formula in [14] does not work in the present case (cf. Cor. 4.6 for the list of indices when $n=3$ ). Here we use a result of [1] to count the number of irreducible solitons. This allows us to show that the list of known solitons constructed in Sect. 3.2 is in fact all the irreducible solitons and hence all irreducible representations of the fixed point subnet (cf. Th. 3.9) can be determined. We expect that this idea will work in other cases. Though our main results Th. 3.9 and Cor. 3.10 are expected from various partial results (cf. [24]), they have not appeared before, and we give some applications of these results.

The rest of this paper is as follows: after preliminary sections on nets and related concepts, we construct solitons using the ideas of [6]. By a counting argument, we prove that all irreps of the fixed point nets have been constructed in Th. 3.9. In Section 4 we consider applications of our main results in Section 3 to the properties of certain fusion matrices which have been studied from different points of view in [18 and 2] where these properties are postulated motivated by considerations from boundary conformal field theories. In Prop. 4.5 we explicitly determine these fusion matrices for the first non-trivial case when $n=3$, using a result in [4] about the set of lines in Euclidean space which mutually have the angles $\pi / 3$ or $\pi / 2$. The result agrees with formulas in [18 and 2]. As a corollary we determine the set of indices of twisted solitons in Cor. 4.6. It is an interesting question to extend these results to $n \geq 4$.

\section{Conformal Nets on $S^{1}$}

In this section we introduce some basic concepts and notations which will be used later. We refer the reader to Section 2 of [14] for more details.

By an interval of the circle we mean an open connected non-empty subset $I$ of $S^{1}$ such that the interior of its complement $I^{\prime}$ is not empty. We denote by $\mathcal{I}$ the family of all intervals of $S^{1}$.

A net $\mathcal{A}$ of von Neumann algebras on $S^{1}$ is a map

$$
I \in \mathcal{I} \rightarrow \mathcal{A}(I) \subset B(\mathcal{H})
$$

from $\mathcal{I}$ to von Neumann algebras on a fixed Hilbert space $\mathcal{H}$ that satisfies:

A. Isotony. If $I_{1} \subset I_{2}$ belong to $\mathcal{I}$, then

$$
\mathcal{A}\left(I_{1}\right) \subset \mathcal{A}\left(I_{2}\right) .
$$

The net $\mathcal{A}$ is called local if it satisfies:

B. Locality. If $I_{1}, I_{2} \in \mathcal{I}$ and $I_{1} \cap I_{2}=\varnothing$, then

$$
\left[\mathcal{A}\left(I_{1}\right), \mathcal{A}\left(I_{2}\right)\right]=\{0\},
$$

where brackets denote the commutator.

The net $\mathcal{A}$ is called Möbius covariant if in addition it satisfies the following properties C, D, E, F:

C. Möbius covariance. There exists a strongly continuous unitary representation $U$ of the Möbius group Möb (isomorphic to $\operatorname{PSU}(1,1)$ ) on $\mathcal{H}$ such that

$$
U(g) \mathcal{A}(I) U(g)^{*}=\mathcal{A}(g I), \quad g \in \text { Möb }, I \in \mathcal{I} .
$$

If $E \subset S^{1}$ is any region, we shall put $\mathcal{A}(E) \equiv \bigvee_{E \supset I \in \mathcal{I}} \mathcal{A}(I)$ with $\mathcal{A}(E)=\mathbb{C}$ if $E$ has empty interior (the symbol $\bigvee$ denotes the von Neumann algebra generated). 
Note that the definition of $\mathcal{A}(E)$ remains the same if $E$ is an interval, namely: if $\left\{I_{n}\right\}$ is an increasing sequence of intervals and $\cup_{n} I_{n}=I$, then the $\mathcal{A}\left(I_{n}\right)$ 's generate $\mathcal{A}(I)$ (consider a sequence of elements $g_{n} \in$ Möb converging to the identity such that $\left.g_{n} I \subset I_{n}\right)$.

D. Positivity of the energy. The generator of the one-parameter rotation subgroup of $U$ (conformal Hamiltonian) is positive.

E. Existence of the vacuum. There exists a unit $U$-invariant vector $\Omega \in \mathcal{H}$ (vacuum vector), and $\Omega$ is cyclic for the von Neumann algebra $\bigvee_{I \in \mathcal{I}} \mathcal{A}(I)$.

By the Reeh-Schlieder theorem $\Omega$ is cyclic and separating for every fixed $\mathcal{A}(I)$. The modular objects associated with $(\mathcal{A}(I), \Omega)$ have a geometric meaning

$$
\Delta_{I}^{i t}=U\left(\Lambda_{I}(2 \pi t)\right), \quad J_{I}=U\left(r_{I}\right) .
$$

Here $\Lambda_{I}$ is a canonical one-parameter subgroup of Möb and $U\left(r_{I}\right)$ is a antiunitary acting geometrically on $\mathcal{A}$ as a reflection $r_{I}$ on $S^{1}$.

This implies Haag duality:

$$
\mathcal{A}(I)^{\prime}=\mathcal{A}\left(I^{\prime}\right), \quad I \in \mathcal{I},
$$

where $I^{\prime}$ is the interior of $S^{1} \backslash I$.

F. Irreducibility. $\bigvee_{I \in \mathcal{I}} \mathcal{A}(I)=B(\mathcal{H})$. Indeed $\mathcal{A}$ is irreducible iff $\Omega$ is the unique $U$-invariant vector (up to scalar multiples). Also $\mathcal{A}$ is irreducible iff the local von Neumann algebras $\mathcal{A}(I)$ are factors. In this case they are $\mathrm{III}_{1}$-factors in Connes classification of type III factors (unless $\mathcal{A}(I)=\mathbb{C}$ for all $I$ ).

By a conformal net (or diffeomorphism covariant net) $\mathcal{A}$ we shall mean a Möbius covariant net such that the following holds:

G. Conformal covariance. There exists a projective unitary representation $U$ of $\operatorname{Diff}\left(S^{1}\right)$ on $\mathcal{H}$ extending the unitary representation of Möb such that for all $I \in \mathcal{I}$ we have

$$
\begin{aligned}
U(g) \mathcal{A}(I) U(g)^{*} & =\mathcal{A}(g I), \quad g \in \operatorname{Diff}\left(S^{1}\right), \\
U(g) x U(g)^{*} & =x, \quad x \in \mathcal{A}(I), g \in \operatorname{Diff}\left(I^{\prime}\right),
\end{aligned}
$$

where Diff $\left(S^{1}\right)$ denotes the group of smooth, positively oriented diffeomorphism of $S^{1}$ and $\operatorname{Diff}(I)$ the subgroup of diffeomorphisms $g$ such that $g(z)=z$ for all $z \in I^{\prime}$.

Let $G$ be a simply connected compact Lie group. By Th. 3.2 of [7], the vacuum positive energy representation of the loop group $L G$ (cf. [19]) at level $k$ gives rise to an irreducible conformal net denoted by $\mathcal{A}_{G_{k}}$. By Th. 3.3 of [7], every irreducible positive energy representation of the loop group $L G$ at level $k$ gives rise to an irreducible covariant representation of $\mathcal{A}_{G_{k}}$.

2.1. Genus 0 S, T-matrices. Next we will recall some of the results of [21] and introduce notations.

Let $\{[\lambda], \lambda \in \mathcal{L}\}$ be a finite set of all equivalence classes of irreducible, covariant, finite-index representations of an irreducible local conformal net $\mathcal{A}$. We will denote the conjugate of $[\lambda]$ by $[\bar{\lambda}]$ and identity sector (corresponding to the vacuum representation) by [1] if no confusion arises, and let $N_{\lambda \mu}^{\nu}=\langle[\lambda][\mu],[\nu]\rangle$. Here $\langle\mu, \nu\rangle$ denotes the dimension of the space of intertwiners from $\mu$ to $v$ (denoted by $\operatorname{Hom}(\mu, v)$ ). We will denote by $\left\{T_{e}\right\}$ a basis of isometries in $\operatorname{Hom}(\nu, \lambda \mu)$. The univalence of $\lambda$ and the statistical dimension of (cf. Section 2 of [11]) will be denoted by $\omega_{\lambda}$ and $d(\lambda)$ (or $\left.d_{\lambda}\right)$ ) respectively. 
Let $\varphi_{\lambda}$ be the unique minimal left inverse of $\lambda$, define:

$$
Y_{\lambda \mu}:=d(\lambda) d(\mu) \varphi_{\mu}\left(\epsilon(\mu, \lambda)^{*} \epsilon(\lambda, \mu)^{*}\right),
$$

where $\epsilon(\mu, \lambda)$ is the unitary braiding operator (cf. [11]).

We list two properties of $Y_{\lambda \mu}$ (cf. (5.13), (5.14) of [21]) which will be used in the following:

\section{Lemma 2.1.}

$$
\begin{gathered}
Y_{\lambda \mu}=Y_{\mu \lambda}=Y_{\lambda \bar{\mu}}^{*}=Y_{\bar{\lambda} \bar{\mu}} . \\
Y_{\lambda \mu}=\sum_{k} N_{\lambda \mu}^{v} \frac{\omega_{\lambda} \omega_{\mu}}{\omega_{\nu}} d(\nu) .
\end{gathered}
$$

We note that one may take the second equation in the above lemma as the definition of $Y_{\lambda \mu}$.

Define $a:=\sum_{i} d_{\rho_{i}}^{2} \omega_{\rho_{i}}^{-1}$. If the matrix $\left(Y_{\mu \nu}\right)$ is invertible, by the proposition on $\mathrm{p} .351$ of [21] $a$ satisfies $|a|^{2}=\sum_{\lambda} d(\lambda)^{2}$.

Definition 2.2. Let $a=|a| \exp \left(-2 \pi i \frac{c_{0}}{8}\right)$, where $c_{0} \in \mathbb{R}$ and $c_{0}$ is well defined mod $8 \mathbb{Z}$.

Define matrices

$$
S:=|a|^{-1} Y, T:=C \operatorname{Diag}\left(\omega_{\lambda}\right)
$$

where

$$
C:=\exp \left(-2 \pi i \frac{c_{0}}{24}\right) \text {. }
$$

Then these matrices satisfy (cf. [21]):

\section{Lemma 2.3.}

$$
\begin{aligned}
S S^{\dagger} & =T T^{\dagger}=\mathrm{id}, \\
S T S & =T^{-1} S T^{-1}, \\
S^{2} & =\hat{C}, \\
T \hat{C} & =\hat{C} T=T,
\end{aligned}
$$

where $\hat{C}_{\lambda \mu}=\delta_{\lambda \bar{\mu}}$ is the conjugation matrix.

Moreover

$$
N_{\lambda \mu}^{v}=\sum_{\delta} \frac{S_{\lambda \delta} S_{\mu \delta} S_{\nu \delta}^{*}}{S_{1 \delta}}
$$

is known as the Verlinde formula.

We will refer to the $S, T$ matrices as defined above as genus 0 modular matrices of $\mathcal{A}$ since they are constructed from the fusion rules, monodromies and minimal indices which can be thought of as genus 0 chiral data associated to a Conformal Field Theory.

The commutative algebra generated by $\lambda$ 's with structure constants $N_{\lambda \mu}^{\nu}$ is called the fusion algebra of $\mathcal{A}$. If $Y$ is invertible, it follows from Lemma 2.3, (3) that any nontrivial irreducible representation of the fusion algebra is of the form $\lambda \rightarrow \frac{S_{\lambda \mu}}{S_{1 \mu}}$ for some $\mu$. 
2.2. The orbifolds. Let $\mathcal{A}$ be an irreducible conformal net on a Hilbert space $\mathcal{H}$ and let $\Gamma$ be a finite group. Let $V: \Gamma \rightarrow U(\mathcal{H})$ be a unitary representation of $\Gamma$ on $\mathcal{H}$. If $V: \Gamma \rightarrow U(\mathcal{H})$ is not faithful, we set $\Gamma^{\prime}:=\Gamma / \operatorname{ker} V$.

Definition 2.4. We say that $\Gamma$ acts properly on $\mathcal{A}$ if the following conditions are satisfied:

(1) For each fixed interval I and each $g \in \Gamma, \alpha_{g}(a):=V(g) a V\left(g^{*}\right) \in \mathcal{A}(I), \forall a \in$ $\mathcal{A}(I)$;

(2) For each $g \in \Gamma, V(g) \Omega=\Omega, \forall g \in \Gamma$.

We note that if $\Gamma$ acts properly, then $V(g), g \in \Gamma$ commutes with the unitary representation $U$ of Möb.

Define $\mathcal{B}(I):=\left\{a \in \mathcal{A}(I) \mid \alpha_{g}(a)=a, \forall g \in \Gamma\right\}$ and $\mathcal{A}^{\Gamma}(I):=\mathcal{B}(I) P_{0}$ on $\mathcal{H}_{0}$, where $\mathcal{H}_{0}:=\{x \in \mathcal{H} \mid V(g) x=x, \forall g \in \Gamma\}$ and $P_{0}$ is the projection from $\mathcal{H}$ to $\mathcal{H}_{0}$. Then $U$ restricts to an unitary representation (still denoted by $U$ ) of Möb on $\mathcal{H}_{0}$. The following is proved in [27]:

Proposition 2.5. The map $I \in \mathcal{I} \rightarrow \mathcal{A}^{\Gamma}(I)$ on $\mathcal{H}_{0}$ together with the unitary representation (still denoted by $U$ ) of Möb on $\mathcal{H}_{0}$ is an irreducible Möbius covariant net.

The irreducible Möbius covariant net in Prop. 2.5 will be denoted by $\mathcal{A}^{\Gamma}$ and will be called the orbifold of $\mathcal{A}$ with respect to $\Gamma$. We note that by definition $\mathcal{A}^{\Gamma}=\mathcal{A}^{\Gamma^{\prime}}$.

2.3. Complete rationality. We first recall some definitions from [16] . Recall that $\mathcal{I}$ denotes the set of intervals of $S^{1}$. Let $I_{1}, I_{2} \in \mathcal{I}$. We say that $I_{1}, I_{2}$ are disjoint if $\bar{I}_{1} \cap \bar{I}_{2}=\varnothing$, where $\bar{I}$ is the closure of $I$ in $S^{1}$. When $I_{1}, I_{2}$ are disjoint, $I_{1} \cup I_{2}$ is called a 1 -disconnected interval in [28]. Denote by $\mathcal{I}_{2}$ the set of unions of disjoint 2 elements in $\mathcal{I}$. Let $\mathcal{A}$ be an irreducible Möbius covariant net as in Section 2.1. For $E=I_{1} \cup I_{2} \in \mathcal{I}_{2}$, let $I_{3} \cup I_{4}$ be the interior of the complement of $I_{1} \cup I_{2}$ in $S^{1}$, where $I_{3}, I_{4}$ are disjoint intervals. Let

$$
\mathcal{A}(E):=A\left(I_{1}\right) \bigvee A\left(I_{2}\right), \quad \hat{\mathcal{A}}(E):=\left(A\left(I_{3}\right) \bigvee A\left(I_{4}\right)\right)^{\prime} .
$$

Note that $\mathcal{A}(E) \subset \hat{\mathcal{A}}(E)$. Recall that a net $\mathcal{A}$ is split if $\mathcal{A}\left(I_{1}\right) \bigvee \mathcal{A}\left(I_{2}\right)$ is naturally isomorphic to the tensor product of von Neumann algebras $\mathcal{A}\left(I_{1}\right) \otimes \mathcal{A}\left(I_{2}\right)$ for any disjoint intervals $I_{1}, I_{2} \in \mathcal{I}$. $\mathcal{A}$ is strongly additive if $\mathcal{A}\left(I_{1}\right) \bigvee \mathcal{A}\left(I_{2}\right)=\mathcal{A}(I)$, where $I_{1} \cup I_{2}$ is obtained by removing an interior point from $I$.

Definition 2.6. [16]. $\mathcal{A}$ is said to be completely rational if $\mathcal{A}$ is split, strongly additive, and the index $[\hat{\mathcal{A}}(E): \mathcal{A}(E)]$ is finite for some $E \in \mathcal{I}_{2}$. The value of the index $[\hat{\mathcal{A}}(E): \mathcal{A}(E)]$ (it is independent of $E$ by Prop. 5 of [16]) is denoted by $\mu_{\mathcal{A}}$ and is called the $\mu$-index of $\mathcal{A}$. If the index $[\hat{\mathcal{A}}(E): \mathcal{A}(E)]$ is infinity for some $E \in \mathcal{I}_{2}$, we define the $\mu$-index of $\mathcal{A}$ to be infinity.

Note that by [17] every irreducible, split, local conformal net with finite $\mu$-index is automatically strongly additive. The following theorem is proved in [27]:

Theorem 2.7. Let $\mathcal{A}$ be an irreducible Möbius covariant net and let $\Gamma$ be a finite group acting properly on $\mathcal{A}$. Suppose that $\mathcal{A}$ is completely rational. Then:

(1) $\mathcal{A}^{\Gamma}$ is completely rational or $\mu$-rational and $\mu_{\mathcal{A}^{\Gamma}}=\left|\Gamma^{\prime}\right|^{2} \mu_{\mathcal{A}}$; 
(2) There are only a finite number of irreducible covariant representations of $\mathcal{A}^{\Gamma}$ (up to unitary equivalence), and they give rise to a unitary modular category as defined in II.5 of [22] by the construction as given in Section 1.7 of [26].

Suppose that $\mathcal{A}$ and $\Gamma$ satisfy the assumptions of Th. 2.7. Then $\mathcal{A}^{\Gamma}$ has only finitely number of irreducible representations $\dot{\lambda}$ and

$$
\sum_{\dot{\lambda}} d(\dot{\lambda})^{2}=\mu_{\mathcal{A} \Gamma}=\left|\Gamma^{\prime}\right|^{2} \mu_{\mathcal{A}} .
$$

2.4. Restriction to the real line: Solitons. Denote by $\mathcal{I}_{0}$ the set of open, connected, non-empty, proper subsets of $\mathbb{R}$, thus $I \in \mathcal{I}_{0}$ iff $I$ is an open interval or half-line (by an interval of $\mathbb{R}$ we shall always mean a non-empty open bounded interval of $\mathbb{R}$ ).

Given a net $\mathcal{A}$ on $S^{1}$ we shall denote by $\mathcal{A}_{0}$ its restriction to $\mathbb{R}=S^{1} \backslash\{-1\}$. Thus $\mathcal{A}_{0}$ is an isotone map on $\mathcal{I}_{0}$, that we call a net on $\mathbb{R}$. In this paper we denote by $J_{0}:=(0, \infty) \subset \mathbb{R}$.

A representation $\pi$ of $\mathcal{A}_{0}$ on a Hilbert space $\mathcal{H}$ is a map $I \in \mathcal{I}_{0} \mapsto \pi_{I}$ that associates to each $I \in \mathcal{I}_{0}$ a normal representation of $\mathcal{A}(I)$ on $B(\mathcal{H})$ such that

$$
\pi_{\tilde{I}} \mid \mathcal{A}(I)=\pi_{I}, \quad I \subset \tilde{I}, \quad I, \tilde{I} \in \mathcal{I}_{0} .
$$

A representation $\pi$ of $\mathcal{A}_{0}$ is also called a soliton. As $\mathcal{A}_{0}$ satisfies half-line duality, namely

$$
\mathcal{A}_{0}(-\infty, a)^{\prime}=\mathcal{A}_{0}(a, \infty), \quad a \in \mathbb{R}
$$

by the usual DHR argument [5] $\pi$ is unitarily equivalent to a representation $\rho$ which acts identically on $\mathcal{A}_{0}(-\infty, 0)$, thus $\rho$ restricts to an endomorphism of $\mathcal{A}\left(J_{0}\right)=\mathcal{A}_{0}(0, \infty)$. $\rho$ is said to be localized on $J_{0}$ and we also refer to $\rho$ as a soliton endomorphism.

Clearly a representation $\pi$ of $\mathcal{A}$ restricts to a soliton $\pi_{0}$ of $\mathcal{A}_{0}$. But a representation $\pi_{0}$ of $\mathcal{A}_{0}$ does not necessarily extend to a representation of $\mathcal{A}$.

If $\mathcal{A}$ is strongly additive, and a representation $\pi_{0}$ of $\mathcal{A}_{0}$ extends to a DHR representation of $\mathcal{A}$, then it is easy to see that such an extension is unique, and in this case we will use the same notation $\pi_{0}$ to denote the corresponding DHR representation of $\mathcal{A}$.

2.5. Induction and restriction. Let $\mathcal{A}$ be a Möbius covariant net and $\mathcal{B}$ a subnet. Given a bounded interval $I_{0} \in \mathcal{I}_{0}$ we fix the canonical endomorphism $\gamma_{I_{0}}$ associated with $\mathcal{B}\left(I_{0}\right) \subset \mathcal{A}\left(I_{0}\right)$. Then we can choose for each $I \in \mathcal{I}_{0}$ with $I \supset I_{0}$ a canonical endomorphism $\gamma_{I}$ of $\mathcal{A}(I)$ into $\mathcal{B}(I)$ in such a way that $\gamma_{I} \uparrow \mathcal{A}\left(I_{0}\right)=\gamma_{I_{0}}$ and $\lambda_{I_{1}}$ is the identity on $\mathcal{B}\left(I_{1}\right)$ if $I_{1} \in \mathcal{I}_{0}$ is disjoint from $I_{0}$, where $\lambda_{I} \equiv \gamma_{I}\lceil\mathcal{B}(I)$.

We then have an endomorphism $\gamma$ of the $C^{*}$-algebra $\mathfrak{A} \equiv \overline{\cup_{I} \mathcal{A}(I)}$ (I bounded interval of $\mathbb{R}$ ).

Given a DHR endomorphism $\rho$ of $\mathcal{B}$ localized in $I_{0}$, the $\alpha$-induction $\alpha_{\rho}$ of $\rho$ is the endomorphism of $\mathfrak{A}$ given by

$$
\alpha_{\rho} \equiv \gamma^{-1} \cdot \operatorname{Ad} \varepsilon(\rho, \lambda) \cdot \rho \cdot \gamma
$$

where $\varepsilon$ denotes the right braiding unitary symmetry (there is another choice for $\alpha$ associated with the left braiding). $\alpha_{\rho}$ is localized in a right half-line containing $I_{0}$, namely $\alpha_{\rho}$ is the identity on $\mathcal{A}(I)$ if $I$ is a bounded interval contained in the left complement 
of $I_{0}$ in $\mathbb{R}$. Up to unitary equivalence, $\alpha_{\rho}$ is localizable in any right half-line, thus $\alpha_{\rho}$ is normal on left half-lines, that is to say, for every $a \in \mathbb{R}, \alpha_{\rho}$ is normal on the $C^{*}$-algebra $\mathfrak{A}(-\infty, a) \equiv \overline{\cup_{I \subset(-\infty, a)} \mathcal{A}(I)}$ ( $I$ bounded interval of $\left.\mathbb{R}\right)$, namely $\alpha_{\rho} \uparrow$ $\mathfrak{A}(-\infty, a)$ extends to a normal morphism of $\mathcal{A}(-\infty, a)$. We have the following Prop. 3.1 of [17]:

Proposition 2.8. $\alpha_{\rho}$ is a soliton endomorphism of $\mathcal{A}_{0}$.

2.6. Loop groups of type A. We denote $\operatorname{LSU}(n)$ the group of smooth maps $f: S^{1} \mapsto$ $S U(n)$ under pointwise multiplication. The diffeomorphism group of the circle Diff $S^{1}$ is naturally a subgroup of $\operatorname{Aut}(L S U(n))$ with the action given by reparametrization. In particular the group of rotations $\operatorname{Rot} S^{1} \simeq U(1)$ acts on $L S U(n)$. The Lie algebra of $L S U(n)$, denoted by $\operatorname{Lsu}(n)$, consists of smooth maps from $S^{1}$ to $s u(n)$. We will denote elements of $L s u(n)$ by its Fourier series $g(z)=\sum_{n} g_{n} z^{n}$, and $L^{0} s u(n)$ the subspace of $\operatorname{Lsu}(n)$ which are polynomials in $z=\exp (2 \pi i \theta), 0 \leq \theta \leq 1$. We will be interested in the projective unitary representation $\pi: L S U(n) \rightarrow U(H)$ that are both irreducible and have positive energy. This means that $\pi$ should extend to $L S U(n) \ltimes \operatorname{Rot} S^{1}$ so that $H=\oplus_{n \geq 0} H(n)$, where the $H(n)$ are the eigenspace for the action of $\operatorname{Rot} S^{1}$, i.e., $r_{\theta} \xi=\exp (\overline{i n} \theta)$ for $\theta \in H(n)$ and $\operatorname{dim} H(n)<\infty$ with $H(0) \neq 0$. By I.7 of [25] the space of finite energy vectors are $C^{\infty}$ vectors for the action of $L^{0} s u(n)$, and by I.9 of [25] $H$ remains irreducible when restricting to subgroups generated by $\exp (i X), X=X^{*} \in L^{0} s u(n)$. We will use $\mathcal{L} S U(n)$ to denote the central extension of $L S U(n)$ by $S^{1}$ as constructed in Chapter 4 of [19].

It follows from [19 and 13] that for fixed level $k$ which is a positive integer, there are only a finite number of such irreducible representations indexed by the finite set

$$
P_{++}^{k}=\left\{\lambda \in P \mid \lambda=\sum_{i=1, \ldots, n-1} \lambda_{i} \Lambda_{i}, \lambda_{i} \geq 0, \sum_{i=1, \ldots, n-1} \lambda_{i} \leq k\right\}
$$

where $P$ is the weight lattice of $S U(n)$ and $\Lambda_{i}$ are the fundamental weights.

We will use $\Lambda_{0}$ or simply 1 to denote the trivial representation of $S U(n)$. For $\lambda, \mu, v \in$ $P_{++}^{k}$, define $N_{\lambda \mu}^{v}=\sum_{\delta \in P_{++}^{k}} S_{\lambda}^{(\delta)} S_{\mu}^{(\delta)} S_{\nu}^{(\delta *)} / S_{\Lambda_{0}}^{(\delta)}$, where $S_{\lambda}^{(\delta)}$ is given by the Kac-Peterson formula (cf. Eq. (4) below for an equivalent formula):

$$
S_{\lambda}^{(\delta)}=c \sum_{w \in S_{n}} \varepsilon_{w} \exp (i w(\delta) \cdot \lambda 2 \pi / n),
$$

where $\varepsilon_{w}=\operatorname{det}(w)$ and $c$ is a normalization constant fixed by the requirement that $S_{\mu}^{(\delta)}$ is an orthonormal system. It is shown in [13], p. 288 that $N_{\lambda \mu}^{v}$ are non-negative integers. Moreover, define $\operatorname{Gr}\left(C_{k}\right)$ to be the ring whose basis are elements of $P_{++}^{k}$ with structure constants $N_{\lambda \mu}^{v}$. The natural involution $*$ on $P_{++}^{k}$ is defined by $\lambda \mapsto \bar{\lambda}=$ the conjugate of $\lambda$ as a representation of $S U(n)$.

We shall also denote $S_{\Lambda_{0}}^{(\Lambda)}$ by $S_{1}^{(\Lambda)}$. Define $d_{\lambda}=\frac{S_{1}^{(\lambda)}}{S_{1}^{\left(\Lambda_{0}\right)}}$. We shall call $\left(S_{\nu}^{(\delta)}\right)$ the $S$-matrix of $\operatorname{LSU}(n)$ at level $k$.

The irreducible positive energy representations of $\operatorname{LSU}(n)$ at level $k$ give rise to an irreducible conformal net $\mathcal{A}$ (cf. [14]) and its covariant representations. 
We will use $\lambda=\left(\lambda_{1}, \ldots, \lambda_{n-1}\right)$ to denote irreducible representations of $\mathcal{A}$ and also the corresponding endomorphism of $M=\mathcal{A}(I)$. Recall from [14] that $\mathcal{A}(I)$ is generated as a von Neumann algebra by $\pi_{k \Lambda_{0}}(f), \forall f \in L S U(n), f\left\lceil I^{\prime}=e\right.$, where $e$ denotes the identity element of $S U(n)$.

All the sectors $[\lambda]$ with $\lambda$ irreducible generate the fusion ring of $\mathcal{A}$.

The following form of the Kac-Peterson formula for the $S$ matrix will be used later:

$$
\frac{S_{\lambda \mu}}{S_{1 \mu}}=\exp \left(\frac{t(\mu+\rho)}{n(k+n)}\right) c h_{\lambda^{\prime}}\left(x_{1}, \ldots, x_{n-1}, 1\right),
$$

where $c h_{\lambda^{\prime}}$ is the character associated with the finite irreducible representation of $S U(n)$ labeled by $\lambda$, and $x_{i}=\exp \left(-2 \pi i \frac{\mu_{i}^{\prime}}{k+n}\right), \mu_{i}^{\prime}=\sum_{i \leq j \leq n-1}\left(\mu_{j}+1\right), 1 \leq i \leq$ $n-1, t(\lambda)=\sum_{1 \leq i \leq n-1} i \lambda_{i}$.

The following result is proved in [25] (see Corollary 1 of Chapter V in [25]).

Theorem 2.9. Each $\lambda \in P_{++}^{(k)}$ has finite index with index value $d_{\lambda}^{2}$. The fusion ring generated by all $\lambda \in P_{++}^{(k)}$ is isomorphic to $\operatorname{Gr}\left(C_{k}\right)$.

In the case of $S U(2)_{k}$, we will label irreducible representations by a half integer called spin $0 \leq i \leq k / 2$. Here are some examples of fusion rules:

$$
\begin{aligned}
& \frac{1}{2} \times i=\left(i-\frac{1}{2}\right) \oplus\left(i+\frac{1}{2}\right), \quad 0 \leq i \leq \frac{k-1}{2} ; \\
& 1 \times i=(i-1) \oplus i \oplus(i+1), \quad 0 \leq i \leq \frac{k-2}{2} .
\end{aligned}
$$

2.7. Twisted loop group. Let $\tau$ be the order two outer automorphism of $S U(n)$ given by $\tau(A)=\bar{A}, \forall A \in S U(n)$ where $\bar{A}$ is the complex conjugate of $A$. On the Lie algebra $s u(n) \tau$ is given by $\tau(X)=-\bar{X}, \forall X \in s u(n)$. (We identify $s u(n)$ with $n \times n$ Hermitian matrices.) It is convenient in this paper to think of the twisted loop group $L_{\tau} S U(n)$ as a subgroup of $\operatorname{LSU}(n)$. We make the following definition:

Definition 2.10. $L_{\tau} S U(n):=\left\{f \in L S U(n), f\left(\theta+\frac{1}{2}\right)=\tau(f(\theta)), 0 \leq \theta \leq \frac{1}{2}\right\}$; $L_{\tau} \operatorname{su}(n):=\left\{f \in \operatorname{Lsu}(n), f\left(\theta+\frac{1}{2}\right)=\tau(f(\theta)), 0 \leq \theta \leq \frac{1}{2}\right\}$.

Let $s u(n)=s o(n) \oplus g_{1}$, where $g_{1}$ is the eigenspace for $\tau$ with eigenvalue -1 . Note that $g_{1}$ is an irreducible representation of $s o(n)$ under the adjoint action (cf. Section 8 of [13]). Assume that $t=t_{0} \oplus t_{1}$, where $t \subset s u(n)$ is the subset of diagonal matrices. Note that both $t_{0}, t_{1}$ are nontrivial subspaces.

The projective irreducible representations of $L_{\tau} S U(n)$ are similar to that of $L S U(n)$. For each fixed positive integer $k$ we again have finitely many projective unitary irreducible representations of $L_{\tau} S U(n) \ltimes S^{1}$. We shall use $\mathcal{L}_{\tau} S U(n)\left(\operatorname{resp} . \mathcal{L}_{\tau} s u(n)\right)$ to denote the central extensions of $L_{\tau} S U(n)$ (resp. $\left.L_{\tau} s u(n)\right)$ so that the projective unitary irreducible representations of $L_{\tau} S U(n) \ltimes S^{1}$ at level $k$ are irreducible representations of $\mathcal{L}_{\tau} S U(n) \ltimes S^{1}$. These representations correspond to irreducible integrable highest weight modules of $\mathcal{L}_{\tau} s u(n)$ at level $k$. We refer the reader to Chapter II of [24] for more details. The following simple observation follows from Chapter 10 of [13]:

Lemma 2.11. The number of irreducible representations of $\mathcal{L}_{\tau} S U(n)$ at level $k$ is equal to the number of irreducible representations $\lambda$ of $\mathcal{L} S U(n)$ at level $k$ such that $\lambda=\bar{\lambda}$. 
Lemma 2.12. Let $\mathcal{B}$ be the subnet of $\mathcal{A}_{S U(n)_{k}}$ such that $\mathcal{B}(I)$ is generated as a von Neumann algebra by $\pi_{k \Lambda_{0}}\left(g \exp (i X) g^{*}\right), \forall g \in \operatorname{Spin}(n), X=X^{*} \in L t, X(z)=0$, $\forall z \in I^{\prime}$. Then $\mathcal{B}=\mathcal{A}$.

Proof. Note that $\mathcal{B}$ is indeed a subnet of $\mathcal{A}$ since the modular group associated with $\mathcal{A}(I)$ preserves $\mathcal{B}(I)$. To show that $\mathcal{B}(I)=\mathcal{A}(I)$ it is enough to show that $\bigvee_{I} \mathcal{B}(I)=$ $\bigvee_{I} \mathcal{A}(I)$. Let $Y=g y g^{*} z^{n}+g y^{*} g^{*} z^{-n} \in \operatorname{Lsu}(n)$ with $y \in t$ and $g \in \operatorname{Spin}(n)$. Then $\pi_{k \Lambda_{0}}(\exp i Y) \in \bigvee_{I} \mathcal{B}(I)$. Since the adjoint actions of $\operatorname{Spin}(n)$ on $g_{0}, g_{1}$ are irreducible, and $t_{0}, t_{1}$ are nontrivial subspaces, it follows that $g_{0}$ (resp. $g_{1}$ ) is the linear span of $g X g^{*}, \forall g \in \operatorname{Spin}(n), X \neq 0 \in t_{0}$ (resp. $g X g^{*}, \forall g \in \operatorname{Spin}(n), X \neq 0 \in t_{1}$ ). Вy Trotter's product formulas (cf. P. 295 of [20]) we conclude that $\pi_{k \Lambda_{0}}(\exp i Y) \in \bigvee_{I} \mathcal{B}(I)$ for any $Y=Y^{*} \in L^{0} s u(n)$. Since $\pi_{k \Lambda_{0}}$ is irreducible as a representation of the group generated by $\exp (i Y), \forall Y=Y^{*} \in L^{0} s u(n)$, the lemma is proved.

\section{Affine Orbifold Nets Associated with Outer Automorphisms}

Let $k$ be a positive integer (level). Unless otherwise stated we will write $\mathcal{A}=\mathcal{A}_{S U(n)_{k}}$. By identifying $\mathbb{R}^{2 n}=(x, y) \rightarrow x+i y \in \mathbb{C}^{n}$, where $x, y$ are column vectors with $n$ real entries, we have the natural inclusion $S U(n)_{k} \subset \operatorname{Spin}(n)_{k}$. Define $J:=\left(I d_{n},-I d_{n}\right) \in$ $S O(2 n)$ and lift it to $\operatorname{Spin}(2 n)$. Note that for $A \in S U(N), J A J=\bar{A}$, and $J . \Omega=\Omega$, where $\Omega$ is the vacuum vector for vacuum representations of $\mathcal{L} \operatorname{Spin}(2 n)_{k}$. It follows that $A d J$ generates a proper $\mathbb{Z}_{2}$ action of $\mathcal{A}_{S U(n)_{k}}$. This is the action corresponding to the outer automorphism $\tau$ of $S U(n)$. Suppose that the vacuum representation of $\mathcal{A}$ decompose as $1 \oplus \sigma$ as representations of $\mathcal{A}^{\mathbb{Z}_{2}}$, where 1 stands for the vacuum representation. Motivated by Lemma 8.3 of [17], we make the following definition:

Definition 3.1. Let $\rho$ be an irreducible soliton of $\mathcal{A}$ which restricts to a DHR representation of $\mathcal{A}^{\mathbb{Z}_{2}}$, and let $\rho_{+}$be an irreducible component of $\rho\left\lceil\mathcal{A}^{\mathbb{Z} 2}\right.$. $\rho$ is called $\tau$-twisted if $\varepsilon\left(\rho_{+}, \sigma\right) \varepsilon\left(\sigma, \rho_{+}\right)=-1$, where $\varepsilon(.,$.$) is the braiding operator (cf. [11]).$

\subsection{Constructions of solitons.}

\section{Definition 3.2.}

$$
\begin{aligned}
L_{\mathbb{R}} S U(n):= & \left\{f \in L S U(n) \mid f(0)=f(1)=e, f^{(n)}(0)=f^{(n)}(1)=0, \forall n \geq 1\right\} ; \\
L_{\mathbb{R}} T_{0}:= & \left\{\exp \left(i f\left(e^{2 \pi i \theta}\right)\right), f=f^{*} \in L t \mid f^{(n)}(0)=f^{(n)}(1)=0, \forall n \geq 0\right\} ; \\
& L_{I} T_{0}:=\left\{\exp (i f), f=f^{*} \in L t, I \subset \mathbb{R}, f\left\lceil I^{\prime}=0\right\} .\right.
\end{aligned}
$$

The following is a special case of the covering homomorphism in Prop. 4.6 of [6]:

Definition 3.3. $\varphi: L_{\mathbb{R}} T_{0} \rightarrow L_{\tau} T$ is a homomorphism defined by $\varphi(g)(\theta)=g(2 \theta)$, $\varphi(g)\left(\theta+\frac{1}{2}\right)=\tau(\varphi(g)(\theta)), 0 \leq \theta \leq 1 / 2$. $\varphi$ lifts to a homomorphism from central extensions of $\mathcal{L}_{R} T_{0}$ to central extensions of $\mathcal{L}_{\tau} T$ and by abuse of notations we will use $\varphi$ to denote the lift. 
3.1.1. Level 1 case. When the level $k=1$, we have the conformal inclusion $\operatorname{Spin}(n)_{2} \subset$ $S U(n)_{1}$, and by Lemma 5.1 of [27] we have $\mathcal{A}^{\mathbb{Z}_{2}}=\mathcal{A}_{\operatorname{Spin}(n)_{2}}$. By 4.3 of [27] $\mathcal{A}_{\operatorname{Spin}(n)_{2}}$ is completely rational, and its irreducible representations are in one to one correspondence to irreducible representations of $\mathcal{L} \operatorname{Spin}(n)_{2}$.

Denote by $T$ the subgroup of diagonal matrices in $S U(n)$ and $\mathcal{L} T$ the subgroup of $\mathcal{L} S U(n)_{1}$. The conformal net $\mathcal{A}_{T}$ associated with $\mathcal{L} T$ is the same as $\mathcal{A}_{S U(n)_{1}}$ by p. 28 of [29], and is a special case of the conformal net associated with lattices as defined in Definition 3.7 of [6]. Hence the $\tau$-twisted irreducible solitons of $\mathcal{A}$ are given by Sect. 4.1 of [6]. These $\tau$-twisted solitons are finitely direct sums of irreducible representations of $\mathcal{L} \operatorname{Spin}(n)_{2}$. (In particular they furnish a representation of $\operatorname{Spin}(n)$ ), and they are in one to one correspondence to irreducible representations of $\mathcal{L}_{\tau} S U(n)$ at level 1 (cf. [3,8] and references therein.) We summarize these results in the following:

Lemma 3.4. The list $\left\{\pi_{\tau}\right\}$ of irreducible $\tau$-twisted solitons of $\mathcal{A}$ is given as follows: they are in one to one correspondence with the list of irreducible representations $\{\pi\}$ of $\mathcal{L}_{\tau} S U(n)$ at level 1 , and we have

$$
\pi_{\tau}\left(g f g^{*}\right)=\pi(g) \pi(\varphi(f)) \pi\left(g^{*}\right), \quad \forall f \in \mathcal{L}_{I} T_{0} \subset \mathcal{L}_{\mathbb{R}} T_{0}, \forall g \in \operatorname{Spin}(n) .
$$

Lemma 3.5. Let $X=g x g^{*} z^{n}+g x^{*} g^{*} z^{-n} \in L_{\sigma} s u(n)$ with $g \in \operatorname{Spin}(n), x \in t$. Then there exists a sequence of $g_{m} \in \mathcal{L}_{\mathbb{R}} S U(n)$ such that

$$
\pi(\exp (i X))=s-\lim _{m \rightarrow \infty} \pi\left(\varphi\left(g_{m}\right)\right),
$$

where $\pi$ is a direct sum of finitely many irreducible representations of $\mathcal{L}_{\tau} S U(n)_{1}$. The same is true if $\pi$ is replaced by $\pi \otimes \pi \ldots \otimes \pi$, where there are $k$ tensor products of $\pi$.

Proof. The proof is similar to the proof of Prop. 4.10 of [6]. By Prop. 1.2.3 in Chapter 4 of [23] we can choose a sequence $x_{m}(\theta)$ of smooth complex valued functions on $[0,1]$ such that $x_{m}\left(\theta+\frac{1}{2}\right)=x_{m}(\theta), x_{m}^{(n)}(0)=x_{m}^{(n)}(1)=0, \forall n \geq 0,\left\|x_{m}-1\right\|_{\frac{1}{2}} \leq 1 / m, m \geq 1$ (cf. Section 1.2 of [23] for the definition of norm $\|.\|_{\frac{1}{2}}$ ). It follows by definition that $\exp \left(i x_{m} X\right)=\varphi\left(\exp \left(i f_{m}\right)\right)$, where $f_{m}(\theta)=x_{m}(\theta / 2) X(\theta / 2), 0 \leq \theta \leq 1$. Note that $\pi$ is a direct sum of finitely many irreducible representations of $\mathcal{L} \operatorname{Spin}(n)_{2}$, by Prop. 1.3.2 in Chapter 4 of [23] we have that $\pi\left(\varphi\left(\exp \left(i f_{m}\right)\right)\right) \rightarrow \pi(\exp (i X))$ strongly. When $\pi$ is replaced by $\pi \otimes \pi \ldots \otimes \pi$, where there are $k$ tensor products of $\pi$, the same argument as in Prop. 1.3.2 in Chapter 4 of [23] works, provided that one replaces the generator $L_{0}$ by $\sum_{1 \leq i \leq k} i d \otimes \ldots \otimes L_{0} \ldots \otimes i d$, where in the summation $L_{0}$ appears in the $i^{t h}$ tensor.

3.2. General level case. Let $\pi_{1}$ be a direct sum of all level 1 irreducible representations of $\mathcal{L}_{\tau} S U(n)$ and let $\pi$ be $k$ tensor products of $\pi_{1}$. Note that $\pi$ is a representation of $\mathcal{L}_{\tau} S U(N)$ at level $k$ with positive energy. By Lemma $3.4 \pi$ gives a soliton of $\mathcal{A}_{S U(n)_{1}}^{\otimes k} \supset$ $\mathcal{A}_{S U(n)_{k}}$, and by restriction, a soliton $\pi_{\tau}$ of $\mathcal{A}_{S U(n)_{k}}$. We have

$$
\pi_{\tau}\left(g f g^{*}\right)=\pi(g) \pi(\varphi(f)) \pi\left(g^{*}\right), \quad \forall f \in \mathcal{L}_{I} T_{0}, I \subset \mathbb{R}, \forall g \in \operatorname{Spin}(n),
$$

where $\varphi$ is defined in Definition 3.3, and we have identified $f$ with its image in $\mathcal{A}(I)$.

Note that $\mathcal{A}_{S U(n)_{1}}^{\otimes k}=\mathcal{A}_{T}^{\otimes k}$ is a net associated with $\mathcal{L}(T \times T \ldots \times T)$, where there are $k$ products, and is a net associated with a lattice as in Definition 3.7 of [6]. By Prop. 4.8 of [6] $\pi$ restricts to a DHR representation of $\left(\mathcal{A}_{S U(n)_{1}}^{\otimes k}\right)^{\mathbb{Z}_{2}}$ where $\mathbb{Z}_{2}$ is generated by $\tau \otimes \tau \ldots \otimes \tau$, hence $\pi_{\tau}$ restricts to a DHR representation of $\mathcal{A}_{S U(n)_{k}}^{\mathbb{Z}_{2}}$. 


\section{Proposition 3.6.}

$$
\left.\bigvee_{I \subset \mathbb{R}} \pi_{\tau}\left(\mathcal{A}_{I}\right)\right)=\pi\left(\mathcal{L}_{\tau} S U(n)\right)^{\prime \prime}
$$

Proof. Since by Lemma $2.12 \mathcal{A}(I)$ is generated by $g f g^{*}, \forall f \in \mathcal{L}_{I} T_{0}, \forall g \in \operatorname{Spin}(n)$, by definition we have

$$
\left.\bigvee_{I \subset \mathbb{R}} \pi_{\tau}\left(\mathcal{A}_{I}\right)\right) \subset \pi\left(\mathcal{L}_{\tau} S U(n)\right)^{\prime \prime}
$$

Since $L_{\tau} S U(n)$ is connected (cf. Lemma 4.2 of [24]), it is sufficient to check that for $X \in L_{\tau} s u(n), \pi(\exp (i X)) \in \bigvee_{I \subset \mathbb{R}} \pi_{\tau}\left(\mathcal{A}_{I}\right)$. As in the proof of Lemma 2.12, by Trotter's product formula (cf. p. 295 of [20]) and irreducibility of the actions of $\operatorname{Spin}(n)$ on $\operatorname{so}(n)$ and $g_{1}$, it is sufficient to check that for $X=g x z^{n} g^{*}+g x^{*} z^{-n} g^{*} \in L_{\sigma} s u(n)$ with $g \in \operatorname{Spin}(n), x \in t$, we have $\pi(\exp (i X)) \in \bigvee_{I \subset \mathbb{R}} \pi_{\tau}\left(\mathcal{A}_{I}\right)$, and this follows from Lemma 3.5.

Corollary 3.7. (1) Each irreducible representation $\rho$ of $\mathcal{L}_{\tau} S U(n)$ at level $k$ gives an irreducible soliton $\rho_{\tau}$ of $\mathcal{A}_{S U(n)_{k}}$ such that $\rho_{1} \simeq \rho_{2}$ as representations of $\mathcal{L}_{\tau} S U(n)$ at level $k$ iff $\rho_{1 \tau} \simeq \rho_{2 \tau}$ as solitons of $\mathcal{A}_{S U(n)_{k}}$;

(2) $\rho_{\tau} \tau \simeq \rho_{\tau}$ as solitons of $\mathcal{A}_{S U(n)_{k}}$, and $\rho_{\tau}$ restricts to a DHR representation of $\mathcal{A}_{S U(n)_{k}}^{\mathbb{Z}_{2}}$

Proof. By [13] all irreducible representations of $\mathcal{L}_{\tau} S U(n)$ at level $k$ appear in $\pi$, and (1) follows from Prop. 3.6. For (2), first we note that $\rho_{\tau}$ comes from an irreducible component of $\pi_{\tau}$ in Prop. 3.6. Since $\pi_{\tau}$ restricts to a DHR representation of $\mathcal{A}_{S U(n)_{k}}^{\mathbb{Z}_{2}}$, it follows that $\rho_{\tau}$ restricts to a DHR representation of $\mathcal{A}_{S U(n)_{k}}^{\mathbb{Z}_{2}}$. By construction

$$
\rho_{\tau}\left(\tau\left(g f g^{*}\right)\right)=\rho\left(\varphi\left(\tau\left(g f g^{*}\right)\right)\right)=\rho\left(R_{1 / 2} \varphi\left(g f g^{*}\right)\right), \quad \forall g \in \operatorname{Spin}(n), f \in \mathcal{L}_{I} T_{0},
$$

where $R_{1 / 2}(g)(\theta)=g\left(\theta+\frac{1}{2}\right)$. Since rotations are implemented on $\rho$ (cf. p. 246 in [13] for a formula for the infinitesimal generator of rotations), (2) follows.

Let $\rho_{\tau}$ be an irreducible soliton of $\mathcal{A}$ as given by Cor. 3.7. By (2) of Cor. 3.9 and Cor. 4.9 of [16] $\rho_{\tau}$ decomposes into a direct sum of two distinct irreducible representations $\rho_{+}, \rho_{-}$of $\mathcal{A}^{\mathbb{Z}_{2}}$. By the same argument as Prop. 4.17 of [6] it follows that $\varepsilon\left(\rho_{+}, \sigma\right) \varepsilon\left(\sigma, \rho_{+}\right)=-1$, and so we have the following lemma:

Lemma 3.8. The irreducible solitons as given in Cor. 3.7 are $\tau$-twisted as in Definition 3.1.

3.3. Counting of all irreducible $\tau$-twisted solitons. Now we apply induction and restriction for general orbifolds in Section 4 of [16] to $\mathcal{A}^{\mathbb{Z}_{2}} \subset \mathcal{A}$. Recall that the vacuum representation of $\mathcal{A}$ decompose as $1 \oplus \sigma$ as representations of $\mathcal{A}^{\mathbb{Z}_{2}}$, where 1 stands for the vacuum representation. Let $\rho_{\tau}$ be a $\tau$-twisted irreducible soliton of $\mathcal{A}$ as given by Cor. 3.7. By (2) of Cor. 3.9 and Cor. 4.9 of [16] $\rho_{\tau}$ decomposes into a direct sum of two distinct irreducible representations $\rho_{+}, \rho_{-}$of $\mathcal{A}^{\mathbb{Z}_{2}}$, and $\left[\alpha_{\rho_{+}}\right]=\left[\alpha_{\rho_{+}}\right]=\left[\rho_{\tau}\right]$. Similarly for an irreducible representation $\lambda$ of $\mathcal{A}$, we have that if $[\lambda]=[\bar{\lambda}]$, then $\lambda$ 
decomposes as a direct sum of two distinct irreducible representations $\lambda_{+}, \lambda_{-}$of $\mathcal{A}^{\mathbb{Z}_{2}}$, and $\left[\alpha_{\lambda_{+}}\right]=\left[\alpha_{\lambda_{-}}\right]=[\lambda]$. If $[\lambda] \neq[\bar{\lambda}]$, then $\lambda$ and $\bar{\lambda}$ restrict to the same representation (denoted by $\lambda$ ) of $\mathcal{A}^{\mathbb{Z}_{2}}$, and we have $\left[\alpha_{\lambda}\right]=[\lambda]+[\bar{\lambda}]$. Denote by $a$ the number of irreducible $\tau$-twisted solitons of $\mathcal{A}, b$ the number of irreducible representations $\lambda$ of $\mathcal{A}$ such that $\lambda=\bar{\lambda}$, and $c$ the number of irreducible representations $\lambda$ of $\mathcal{A}$ such that $\lambda \neq \bar{\lambda}$. By Th. 4.16 of [1], we have

$$
a+b+c=2 b+c .
$$

Hence $a=b$. Note that by Lemma 2.11 the number of irreducible representations of $\mathcal{L}_{\tau} S U(n)$ at level $k$ is $b$. By Lemma 3.8 it follows that Cor. 3.7 gives all irreducible $\tau$-twisted representations of $\mathcal{A}$. We summarize these results in the following:

Theorem 3.9. (1) $\rho_{\tau}$ as given in Cor. 3.7 gives all the irreducible $\tau$-twisted representations of $\mathcal{A}$. These representations are in one to one correspondence with irreducible representations of $\mathcal{L}_{\tau} S U(n)$ at level $k$; (2) The list of all irreducible representations of $\mathcal{A}^{\mathbb{Z}_{2}}$ are as follows:

For an irreducible representation $\lambda$ of $\mathcal{A}$, we have that if $[\lambda]=[\bar{\lambda}]$, then $\lambda$ decomposes as a direct sum of two distinct irreducible representations $\lambda_{+}, \lambda_{-}$of $\mathcal{A}^{\mathbb{Z}_{2}}$; If $[\lambda] \neq[\bar{\lambda}]$, then $\lambda$ and $\bar{\lambda}$ restrict to the same representation (denoted by $\lambda$ ) of $\mathcal{A}^{\mathbb{Z}_{2}} ; \rho_{+}, \rho_{-}$where $\rho_{\tau}$ corresponds to irreducible representations of $\mathcal{L}_{\tau} S U(n)$ at level $k$.

By Th. 2 and Th. 3.9, we have proved the following:

Corollary 3.10. The list of irreducible representations of $\mathcal{A}^{\mathbb{Z}_{2}}$ as in (2) of Th. 3.9 give rise to a unitary modular tensor category as defined in II.5 of [22] by the construction as given in Section 1.7 of [26].

\section{Examples of Fusion Rules}

Theorem 3.9 and Corollary 3.10 give strong constraints on the fusion rules related to $\mathcal{A}^{\mathbb{Z}_{2}}$. In this section we give some examples of fusion rules by using the results of Section 2. The ideas are similar to Sect. 9 of [14].

Denote by $N_{\lambda \rho_{1}}^{\rho_{2}}=\left\langle\lambda \rho_{1}, \rho_{2}\right\rangle$, where $\lambda$ is an irreducible representation of $\mathcal{A}$, and $\rho_{1}, \rho_{2}$ are irreducible $\tau$-twisted representations of $\mathcal{A}$.

Lemma 4.1. There exists a complex valued matrix $\psi_{\rho}^{(\mu)}$, where $\rho$ denotes irreducible $\tau$-twisted representations of $\mathcal{A}$, and $\mu=\bar{\mu}$ labels an irreducible representation of $\mathcal{A}$, such that

$$
N_{\lambda \rho_{1}}^{\rho_{2}}=\sum_{\mu, \mu=\bar{\mu}} \frac{S_{\lambda \mu}}{S_{1 \mu}} \psi_{\rho_{1}}^{(\mu)} \psi_{\rho_{2}}^{(\mu) *}
$$

Proof. First we assume that $\lambda=\bar{\lambda}$. We have

$$
\left\langle\lambda \rho_{1}, \rho_{2}\right\rangle=\left\langle\lambda \alpha_{\rho_{1+}}, \alpha_{\rho_{2+}}\right\rangle=\left\langle\left(\lambda_{+}+\lambda_{-}\right) \rho_{1+}, \rho_{2+}\right\rangle .
$$

Using the Verlinde formula we have:

$$
\left\langle\left(\lambda_{+}+\lambda_{-}\right) \rho_{1+}, \rho_{2+}\right\rangle=\sum_{\dot{\mu}}\left(\frac{S_{\lambda_{+} \dot{\mu}} S_{\rho_{1+} \dot{\mu}} S_{\rho_{2+} \dot{\mu}}^{*}}{S_{1 \dot{\mu}}^{3}}+\frac{S_{\lambda_{-} \dot{\mu}} S_{\rho_{1+} \dot{\mu}} S_{\rho_{2+} \dot{\mu}}^{*}}{S_{1 \dot{\mu}}^{3}}\right) .
$$


By Lemma 9.1 of [14], we have that $S_{\rho_{+} \dot{\mu}}=0$ if $\dot{\mu}$ comes from restriction of the representation $\mu \neq \bar{\mu}$ of $\mathcal{A}$, and $S_{\lambda_{+} \dot{\mu}}=-S_{\lambda_{-} \dot{\mu}}$ if $\dot{\mu}$ comes from restriction of a $\tau$-twisted representation of $\mathcal{A}$. If $\mu=\bar{\mu}$, by (4) of Lemma 9.1 in [14] we have

$$
\frac{S_{\lambda_{+} \mu_{+}} S_{\rho_{1+} \mu_{+}} S_{\rho_{2+} \mu_{+}}^{*}}{S_{1 \mu_{+}}^{3}}=\frac{S_{\lambda_{-} \mu_{-}} S_{\rho_{1+} \mu_{-}} S_{\rho_{2+} \mu_{-}}^{*}}{S_{1 \mu_{-}}^{3}}=\frac{S_{\lambda \mu} S_{\rho_{1+} \mu_{+}} S_{\rho_{2+} \mu_{+}}^{*}}{S_{1 \mu} S_{1 \mu_{+}}^{2}} .
$$

Set $\psi_{\rho}^{(\mu)}=\sqrt{2} \frac{S_{\rho_{+} \mu_{+}}}{S_{1 \mu_{+}}}$and the lemma follows. The case when $\lambda \neq \bar{\lambda}$ is similar.

4.1. $n=3$ case. Lemma 4.1 determines the spectrum of the square matrix $N_{\lambda}$ whose entries are non-negative integers. In this section we determine (up to permutation) $N_{\lambda}$ for the first non-trivial case $n=3$. Our results agree with the ansatz given by [18] based on heuristic arguments.

Lemma 4.2. $d=\left(d_{\rho}\right)_{\rho}$ is the unique (up to scalar multiplication) Perron-Frobenius eigenvector of $N_{v}$ with eigenvalue $S_{v 1} / S_{11}$, where $v$ denotes the vector representation.

Proof. We note that the matrix $N_{v}$ is irreducible. In fact since $\rho_{1} \bar{\rho}_{2} \succ \lambda$ for some $\lambda$, and $\lambda \prec v^{m}$ for some integer $m$, it follows that the $\left(\rho_{1}, \rho_{2}\right)^{t h}$ entry of $N_{v}$ is positive. Hence by [9] the lemma is proved.

We shall refer to the equation

$$
N_{v} d=\frac{S_{v 1}}{S_{11}} d
$$

as the Perron-Frobenius equation.

Note that by Lemma $4.1, N_{v}=N_{\bar{v}}$. Since every irrep $\lambda$ can be written as polynomials in $v, \bar{v}$, it is sufficient to determine $N_{v}$.

Let $M=N_{v}-I$. By Lemma 4.1 and Eq. (4) the spectrum of $M$ is given as follows. If $k=2 m-1$ is odd, then the spectrum of $M$ is given by $2 \cos \left(\frac{\pi(i+1)}{m+1}\right), 0 \leq i \leq m-1$, and it is the same as the fusion matrix $N_{1 / 2}$ associated with $S U(2)_{m-1}$, where $1 / 2$ denotes the spin $1 / 2$ representation. If $k=2 m-2$ is even, then the spectrum of $N_{v}$ is given by $2 \cos \left(\frac{2 \pi(i+1)}{2 m+1}\right), 0 \leq i \leq m-1$, and it is the same as the fusion matrix $N_{1}$ associated with $S U(2)_{2 m-1}$ acting on the set of integer spin representations of $S U(2)_{2 m-1}$, where 1 denotes the spin 1 representation of $S U(2)_{2 m-1}$.

Our goal in this section is to show that up to permutation $N_{v}=N_{1 / 2}+I$ when $k=2 m-1$ and $N_{v}=N_{1}$ when $k=2 m-2$.

First note that since $\|M\|<2$, the entries of $M$ can take only $1,-1,0$, and since $M=N_{v}-I$, only diagonal entries on $M$ can be -1 . By the known spectrum of $M$ we have $\operatorname{tr}(M)=0, \operatorname{tr}\left(M^{2}\right)=2 m-2$ when $k=2 m-1$, and $\operatorname{tr}(M)=-1, \operatorname{tr}\left(M^{2}\right)=2 m-1$ when $k=2 m-2$.

Also since $\left\|M^{2}\right\|<4$ each row of $M$ contains at most three nonzero entries.

Denote by $k_{1}, k_{2}, k_{3}$ respectively the number of rows of $M$ with one, two, three nonzero entries respectively. Then we have $k_{1}+k_{2}+k_{3}=n, k_{1}+2 k_{2}+3 k_{3}=\operatorname{tr}\left(M^{2}\right)=$ $2 m-2$ when $k=2 m-1$, and $k_{1}+k_{2}+k_{3}=n, k_{1}+2 k_{2}+3 k_{3}=\operatorname{tr}\left(M^{2}\right)=2 m-1$ when $k=2 m-2$.

Hence $k_{1}=k_{3}+2$ when $k$ is odd and $k_{1}=k_{3}+1$ when $k$ is even. For simplicity we enumerate the $\tau$-twisted solitons by $1, \ldots, m$ We associate a graph $G$ to these solitons 
with vertices $1, \ldots, m$ and connect $i$ and $j(i \neq j)$ by the $(i, j)^{\text {th }}$ entry of $M$. By Lemma $4.2 G$ is connected.

If $k_{3}=0$, then $k_{1}=2$ or $k_{1}=1$ depending on if $k$ is odd or even. Permute solitons if necessary, we may assume that the first row contains only one nonzero entry. Using Eq. (7) we must have 1 in the first row of $M$, and it is not on the diagonal. Assume that 2 is the vertex connected to 1 on $G$ and use Eq. 7 and the fact that $G$ is connected; we conclude that unless $m=2,2$ is connected to a new vertex 3. Continue this argument we have shown that up to permutation $N_{v}=N_{1 / 2}+I$ when $k$ is odd, and $N_{v}=N_{1}$ when $k$ is even.

When $k$ is odd and if all diagonal entries of $M$ are zeros, then $2 I-M$ is a positive definite matrix with all diagonal entries equal to 2 . Hence we can find a basis $\left\{\epsilon_{1}, \ldots \epsilon_{m}\right\}$ in $\mathbb{R}^{m}$ such that $\left(\epsilon_{i}, \epsilon_{j}\right)=0$ or -1 if $i \neq j$ and $\left(\epsilon_{i}, \epsilon_{i}\right)=2,1 \leq i \leq m$. It follows that $G$ is a connected Coexter graph, and $G$ must be $A-D-E$ graph (cf. p. 60 of [12] or Section 1.4 of [10]). Since $G$ has norm $2 \cos \left(\frac{\pi}{m+1}\right)$ with $m$ vertices, by inspecting Table 1.4.5 of [10] we conclude that up to permutation $M=N_{1 / 2}$.

When $k$ is even, $\operatorname{tr}(M)=-1$, there is at least one -1 on the diagonal of $M$.

For the rest of this section we assume that $k_{3}>0$ and there is at least one nonzero entry on the diagonal of $M$. Since one can easily determine $M$ for $m \leq 3$, we will also assume that $m \geq 4$. We will derive contradictions from these assumptions.

The basic idea is contained in Remark (2) on p. 23 of [10]. Introduce new rows numbered by $1^{\prime}, 2^{\prime}, \ldots, m^{\prime}$ and we use $M_{1}$ to denote a symmetric $2 m \times 2 m$ matrix whose $\left(i, j^{\prime}\right)^{\text {th }}$ entry is the $(i, j)^{\text {th }}$ entry of $M$, and all other entries of $M_{1}$ are equal to zero. We associate a graph $G_{1}$ to $M_{1}$ whose vertices are $1,2, \ldots, m, 1^{\prime}, 2^{\prime}, \ldots, m^{\prime}$ and $i, j^{\prime}$ are connected by the absolute value of the $(i, j)^{\text {th }}$ entry of $M$. Since $G$ is connected, and by our assumption there is at least one nonzero entry on the diagonal of $M$, it follows that $G_{1}$ is connected. Let $P=2 I-M_{1}$. Note that $P$ is positive definite, and we can find a basis $\varepsilon_{1}, \ldots \varepsilon_{m}, \varepsilon_{1}^{\prime}, \ldots \varepsilon_{m}^{\prime}$ in $\mathbb{R}^{2 m}$ such that the inner product matrix of this basis is $P$. By definition $\varepsilon_{1}, \ldots, \varepsilon_{m}$ and $\varepsilon_{1}^{\prime}, \ldots, \varepsilon_{m}^{\prime}$ are two orthogonal sets, and the angles between the lines spanned by the elements in the basis are either $\pi / 3$ or $\pi / 2$, and by Th. 3.5 of [4] we conclude that $\varepsilon_{1}, \ldots, \varepsilon_{m}, \varepsilon_{1}^{\prime} \ldots, \varepsilon_{m}^{\prime}$ is contained in a direct sum of root systems of $A-D-E$. Since $G_{1}$ is connected, we conclude that $\varepsilon_{1}, \ldots \varepsilon_{m}, \varepsilon_{1}^{\prime}, \ldots \varepsilon_{m}^{\prime}$ is contained in one root system. If this root system is $E_{6}$ or $E_{8}$, then we have $m=3$ or $m=4$. One can easily rule out these two cases using $k_{1}=k_{3}+2$ or $k_{1}=k_{3}+1$ and Eq. (7). If this root system is $A_{2 m+1}$, since the elements of this system are of the form $e_{i}-e_{j}, 1 \leq i, j \leq 2 m+1$ (cf. Definition 3.1 of [4]), it follows that $M$ can not have a row with three nonzero entries. Hence to finish the proof we assume that $\varepsilon_{1}, \ldots \varepsilon_{m}, \varepsilon_{1}^{\prime}, \ldots \varepsilon_{m}^{\prime}$ is contained in the root system of $D_{2 m}$. The root system of type $D_{2 m}$ consists of vectors $\pm e_{i} \pm e_{j}, 1 \leq$ $i \neq j \leq 2 m$, where $e_{i}, 1 \leq i \leq 2 m$ is an orthonormal basis in $\mathbb{R}^{2 m}$. By assumption we can identify $\varepsilon_{1}, \ldots \varepsilon_{m}, \varepsilon_{1}^{\prime}, \ldots \varepsilon_{m}^{\prime}$ as a subset of $\pm e_{i} \pm e_{j}, 1 \leq i \neq j \leq$ $2 m$.

The following lemma will be used repeatedly in the following, and its proof follows directly from definitions:

Lemma 4.3. (1) If a vertex on $G_{1}$ is connected to three different vertices $i_{1}^{\prime}, i_{2}^{\prime}, i_{3}^{\prime}$, then $\varepsilon_{i_{1}}^{\prime}, \varepsilon_{i_{2}}^{\prime}, \varepsilon_{i_{3}}^{\prime}$ must contain (up to multiplication by -1 ) $e_{j}+e_{k}, e_{j}-e_{k}$ for some $1 \leq j \neq k \leq 2 m$

(2) If two vertices $i^{\prime}, j^{\prime}$ of $G_{1}$ are such that $\varepsilon_{i}^{\prime}=e_{j}+e_{k}, \varepsilon_{j}^{\prime}=e_{j}-e_{k}$, then $i^{\prime}$ is connected to vertex $l$ in $G_{1}$ iff $j^{\prime}$ is connected to vertex $l$;

(3) If $\varepsilon_{i_{1}}^{\prime}=e_{j}+e_{k}, \varepsilon_{i_{2}}^{\prime}=e_{j}-e_{k}, \varepsilon_{j_{1}}=e_{p}+e_{q}, \varepsilon_{j_{2}}=e_{p}-e_{q}$, then $\{j, k\} \cap\{p, q\}=\varnothing$. 
Definition 4.4. A vertex $i$ of $G$ is called type 0 if the $(i, i)^{\text {th }}$ entry of $M$ is zero, and type 1 otherwise.

Case (1). If a type 1 vertex is connected to two different type 0 vertices on $G$, by Lemma 4.3 the two type 0 vertices are not connected. By permuting basis elements if necessary, we may assume that $\left(\varepsilon_{i}, \varepsilon_{j}^{\prime}\right)=0,\left|\left(\varepsilon_{i}, \varepsilon_{i}^{\prime}\right)\right|=1,1 \leq i \neq j \leq 3$. Since $\varepsilon_{1}, \ldots, \varepsilon_{m}, \varepsilon_{1}^{\prime}, \ldots, \varepsilon_{m}^{\prime}$ is contained in the root system of $D_{2 m}, \varepsilon_{1}^{\prime}, \ldots \varepsilon_{m}^{\prime}$ and $\varepsilon_{1}, \ldots, \varepsilon_{m}$ are two orthogonal sets, it follows that we may assume that (up to multiplication by -1 ) $\varepsilon_{1}^{\prime}=e_{3}+e_{5}, \varepsilon_{2}^{\prime}=e_{1}+e_{2}, \varepsilon_{3}^{\prime}=e_{1}-e_{2}$ and $\varepsilon_{1}=e_{1}+e_{5}, \varepsilon_{2}=e_{3}+e_{4}, \varepsilon_{3}=e_{3}-e_{4}$. But then $e_{5}$ is in the subspace spanned by $\varepsilon_{1}^{\prime}, \varepsilon_{2}, \varepsilon_{3}$ and also in the subspace spanned by $\varepsilon_{1}, \varepsilon_{2}^{\prime}, \varepsilon_{3}^{\prime}$, contradicting the fact that $\varepsilon_{1}, \ldots \varepsilon_{m}, \varepsilon_{1}^{\prime}, \ldots \varepsilon_{m}^{\prime}$ is a basis.

Case (2). If two type 1 vertices are connected on $G$, assume that one such vertex is connected to either a type 0 or type 1 vertex. The first case is ruled out by Lemma 4.3 and Case (1), and the second case is impossible by Lemma 4.3 and the fact that $G$ is connected and $m \geq 4$.

Case (3). If two type 1 vertices are connected to the same type 0 vertex on $G$, since $m \geq 4$ and $G$ is connected, by case (1) and (2) we assume that the type 0 vertex is connected to one additional vertex on $G$, but one checks easily that this is impossible by Lemma 4.3 .

Now consider a subgraph $G^{\prime}$ obtained from $G$ by deleting type 1 vertices and edges with one endpoint a type 1 vertex. Since $G$ is connected, by Case (1) $G^{\prime}$ is connected. Moreover, if $i$ is a type 0 vertex, define $\eta_{i}:=\frac{1}{\sqrt{2}}\left(\varepsilon_{i}+\varepsilon_{i}^{\prime}\right)$. Then $\left(\eta_{i}, \eta_{i}\right)=2$, and it follows that two vertices $i_{1}, i_{2}$ on $G^{\prime}$ are connected by $-\left(\eta_{i}, \eta_{j}\right)$ edges. Hence $G^{\prime}$ is a connected Coexter graph, and by p. 60 of [12] we know that $G^{\prime}$ is an $A-D-E$ graph. Since we assume that $k_{3} \geq 1, G^{\prime}$ must be type $D$ or $E$. In the case $k_{1}=k_{3}+2$, we must have no type 1 vertex attached to the end points of $G^{\prime}$, contradicting our assumption that a type 1 vertex exists and must be connected to one type 0 vertex by Case (2). In the case $k_{1}=k_{3}+1$, we must have exactly one type 1 vertex connected to one endpoint of either type $D$ or type $E$ graph, and these cases can be directly ruled out by tedious calculations using the Perron-Frobenius equation. Here we give a different approach. By fusion rules of $S U(2)_{2 m-1}$ in Eq. (5), $N_{m-1}$ can be written as a polynomial of $N_{1}$ with integer coefficients, and we have $N_{m-1}^{2}=N_{1}+I$. The spectrum of $N_{m-1}$ is given by $2 \cos \left(\frac{\pi(i+1)}{2 m+1}\right), 0 \leq i \leq m-1$. Since $N_{1}$ has the same spectrum as $N_{v}$, it follows that there is a symmetric matrix $M^{\prime}$ with integer entries such that $M^{\prime 2}=N_{v}+I$, and $M^{\prime}$ has the same spectrum as $N_{m-1}$. Since $N_{v}$ has one 1 and $m-12$ 's on the diagonal, it follows that $M^{\prime}$ has one row with one nonzero entry \pm 1 , and $m-1$ rows with two nonzero entries which are \pm 1 . Now associate a graph with vertices $1, \ldots, m$ to $M^{\prime}$ so that the $i^{\text {th }}$ and the $j^{\text {th }}$ vertex are connected by the absolute value of the $(i, j)^{\text {th }}$ entry of $M^{\prime}$ (If $i=j$ and the $(i, i)^{\text {th }}$ entry of $M^{\prime}$ is \pm 1 , we connect $i$ to itself by a loop.) As in the case with $M$ this graph is connected, and it follows that it is a line segment with one loop attached to an endpoint. Since $G^{\prime}$ has a trivalent vertex, it follows that there exist four different vertices $i, j, k, l$ such that the $(i, j),(i, k),(i, l)$ entries of $N_{v}$ are 1 , but one checks easily that this is impossible since $M^{\prime 2}=N_{v}+I$. We have proved the following:

Proposition 4.5. (1) When $k=2 m-1$, we can label the $\tau$-twisted irrep of $\mathcal{A}$ by integers $1,2, \ldots, m$ such that the $(i, j)^{\text {th }}$ entry of $N_{v}$ is given by $\delta_{i j}+\left\langle\frac{1}{2} \frac{(i-1)}{2}, \frac{(j-1)}{2}\right\rangle$, where half integers $0 \leq k / 2 \leq m-1 / 2$ label the spin of irreps of $S U(2)_{m-1}$; 
(2) When $k=2 m-2$, we can label the $\tau$-twisted irrep of $\mathcal{A}$ by integers $1,2, \ldots, m$ such that the $(i, j)^{\text {th }}$ entry of $N_{v}$ is given by $\delta_{i j}+\langle 1(i-$ $1),(j-1)\rangle$, where integers $0 \leq k \leq m-1$ label the spin of irreps of $S U(2)_{2 m-1}$.

By Prop. 4.5 the Perron-Frobenius eigenvector $d$ is up to multiplication by a positive constant $\delta$ and possible permutations equal to $\left.\left(d_{(i-1) / 2}\right)_{1 \leq i \leq m}\left(\operatorname{resp} .\left(d_{i-1}\right)_{1 \leq i \leq m}\right)\right)$ when $k=2 m-1$ (resp. when $k=2 m-2$ ). By Prop. 3.1 of [1] $\sum_{\rho_{\tau}} d_{\rho_{\tau}}^{2}=\sum_{\lambda} d_{\lambda}^{2}$, and by Eq. (4) we can determine $\delta$ uniquely. When $k=2 m-1, \delta=\frac{\sqrt{3(m+1)}}{2 \sin ^{2}\left(\frac{\pi}{2 m+2}\right)}$, and when $k=2 m-2, \delta=\frac{\sqrt{3(2 m+1)}}{4 \sin \left(\frac{\pi}{2 m+1}\right) \sin \left(\frac{2 \pi}{2 m+1}\right)}$. We have therefore proved the following

Corollary 4.6. (1) When $k=2 m-1$, the set of indices of $\tau$-twisted solitons are given by $\left\{\frac{3(m+1)}{4 \sin ^{4}\left(\frac{\pi}{2 m+2}\right)} \frac{\sin (i \pi /(m+1))}{\sin (\pi /(m+1))}, 1 \leq i \leq m\right\}$;

(2) When $k=2 m-2$, the set of indices of $\tau$-twisted solitons are given by $\left\{\frac{3(2 m+1)}{16 \sin ^{2}\left(\frac{\pi}{2 m+1}\right) \sin ^{2}\left(\frac{2 \pi}{2 m+1}\right)} \frac{\sin ((2 i-1) \pi /(2 m+1))}{\sin (\pi /(2 m+1))}, 1 \leq i \leq m\right\}$.

Acknowledgement. The author would like to thank Prof. V. G. Kac for stimulating discussions and providing references and useful suggestions. The paper would not have been written without his help.

Open Access This article is distributed under the terms of the Creative Commons Attribution Noncommercial License which permits any noncommercial use, distribution, and reproduction in any medium, provided the original author(s) and source are credited.

\section{References}

1. Böckenhauer, J., Evans, D.E., Kawahigashi, Y.: Chiral structure of modular invariants for subfactors. Commun. Math. Phys. 210(3), 733-784 (2000)

2. Birke, L., Fuchs, J., Schweigert, C.: Symmetry breaking boundary conditions and WZW orbifolds. Adv. Theor. Math. Phys. 3, 671-726 (1999)

3. Bakalov, B., Kac, V.G.: Twisted modules over lattice vertex algebras. In: Lie theory and its applications in physics V, eds Doebner, H.-D., Dobrev. V.K., River Edge, NJ: World Sci. World Sci. Publ., 2004, pp. 3-26

4. Cameron, P.J., Goethals, J.-M., Seidel, J.J., Shult, E.E.: Line graphs, root systems, and elliptic geometry. J. Algebra 43(1), 305-327 (1976)

5. Doplicher, S., Haag, R., Roberts, J.E.: Local observables and particle statistics. I. Commun. Math. Phys. 23, 199-230 (1971); II. 35, 49-85 (1974)

6. Dong, C., Xu, F.: Conformal nets associated with lattices and their orbifolds. Adv. Math. 206(1), 279-306 (2006)

7. Gabbiani, F., Fröhlich, J.: Operator algebras and conformal field theory. Commun. Math. Phys. 155, 569-640 (1993)

8. ten Kroode, F., van de Leur, J.: Level one representations of the twisted affine algebras $A_{n}^{(2)}$ and $D_{n}^{(2)}$. Acta Appl. Math. 27(3), 153-224 (1992)

9. Gantmacher, F.R.: The theory of matrices. Vol. 2. Newyork: Chelsea, 1960

10. Goodman F., de la Harpe, P., Jones, V.F. R.: Coxeter graphs and towers of algebras. Mathematical Sciences Research Institute Publications, 14. New York: Springer-Verlag, 1989

11. Guido, D., Longo, R.: Relativistic invariance and charge conjugation in quantum field theory. Commun. Math. Phys. 148, 521-551 (1992)

12. Humphreys, J.: Introduction to Lie algebras and representation theory. GTM 9. Berline-Heidelberg- New York: Springer-Verlag, 1972

13. Kac V.G.: Infinite Dimensional Lie Algebras. 3rd Edition. Cambridge: Cambridge University Press, 1990

14. Kac, V., Longo, R., Xu, F.: Solitons in affine and permutation orbifolds. Commun. Math. Phys. 253(3), 723764 (2005)

15. Kac, V.G., Wakimoto, M.: Modular and conformal invariance constraints in representation theory of affine algebras. Adv. in Math. 70, 156-234 (1988) 
16. Kawahigashi, Y., Longo, R., Müger, M.: Multi-interval subfactors and modularity of representations in conformal field theory. Commun. Math. Phys. 219, 631-669 (2001)

17. Longo, R., Xu, F.: Topological sectors and a dichotomy in conformal field theory. Commun. Math. Phys. 251, 321-364 (2004)

18. Petkova, V.B., Zuber, J.-B.: Boundary conditions in charge conjugate sl(N) WZW theories. http://arxiv. org/abs/hep-th/0201239v4, 2002

19. Pressley, A., Segal, G.: Loop Groups. Oxford: Oxford University Press, 1986

20. Reed, M., Simon, B.: Methods of modern mathematical physics, I:Functional analysis. Newyork: Academic Press, 1980

21. Rehren, K.-H.: Braid group statistics and their superselection rules. In: The Algebraic Theory of Superselection Sectors. D. Kastler, ed., Singapore: World Scientific, 1990

22. Turaev, V.G.: Quantum invariants of knots and 3-manifolds. Berlin, New York: Walter de Gruyter, 1994

23. Toledano Laredo, V.: Fusion of Positive Energy Representations of LSpin $2 n$. Ph.D. dissertation, University of Cambridge, 1997

24. Verrill, R.: Positive energy representations of $L^{\sigma} S U(2 r)$ and orbifold fusion. Ph.D. dissertation, University of Cambridge, 2001

25. Wassermann, A.: Operator algebras and Conformal field theories III. Invent. Math. 133, 467-538 (1998)

26. Xu, F.: 3-manifold invariants from cosets. J. Knot Theory Ram 14(1), 21-90 (2005)

27. Xu, F.: Algebraic orbifold conformal field theories. PNAS. USA 97(26) 14069-14073, 2000

28. Xu, F.: Jones-Wassermann subfactors for disconnected intervals. Commun. Cont. Math. 2, 307-347 (2000)

29. Xu, F.: Algebraic coset conformal field theories. Commun. Math. Phys. 211, 1-43 (2000)

Communicated by Y. Kawahigashi 